Case Report

\title{
Unusual Complication following a Myomectomy: Colic Migration of a Forgotten Abdominal Swab
}

\author{
Boyodi Tchangai, ${ }^{1}$ Fousseni Alassani, ${ }^{1}$ and Mazamesso Tchaou ${ }^{2}$ \\ ${ }^{1}$ Department of Surgery, Teaching Hospital of Sylvanus Olympio, Lome, Togo \\ ${ }^{2}$ Department of Radiology, Teaching Hospital of Sylvanus Olympio, Lome, Togo \\ Correspondence should be addressed to Boyodi Tchangai; botchangai@yahoo.fr
}

Received 5 November 2016; Revised 21 December 2016; Accepted 9 January 2017; Published 26 January 2017

Academic Editor: Cheng-Yu Long

Copyright (c) 2017 Boyodi Tchangai et al. This is an open access article distributed under the Creative Commons Attribution License, which permits unrestricted use, distribution, and reproduction in any medium, provided the original work is properly cited.

Surgical sponges are the most common retained foreign bodies following surgery. The morbidity of this condition is illustrated herein with the case of a 36-year-old female patient with a history of myomectomy 5 months before her admission into our unit for enterocutaneous fistula. Although imaging and etiological investigations were made, diagnosis was carried out only by laparotomy. The foreign body found was an abdominal swab that migrated from abdominal cavity to the colon causing several intestinal injuries. The lack of specific clinical signs and the death of the patient raise the necessity of preventing these complications that involve the surgeon liability.

\section{Introduction}

Surgical sponges are the most common retained foreign bodies following surgery [1]. They are known as textiloma or gossypiboma and occur with various surgical procedures including abdominal surgery. Textiloma results in an inflammatory reaction that becomes an encapsulated fibroblastic granuloma [2]. The clinical consequences are unpredictable and potentially disastrous. Enteric migration of abdominal textiloma is a rare complication that brings about considerable morbidity $[1,3]$. We report a case of intracolic migration of an abdominal swab mistakenly left behind after myomectomy that resulted in death. The diagnosis difficulties and the importance of prevention are highlighted.

\section{Case Description}

A 36-year-old female patient was sent to our emergency unit for a subocclusive syndrome and enterocutaneous fistula. She has had a myomectomy in another health centre 6 months ago. On the 29th postoperative day, she experienced disorders with severe colicky abdominal pain and intermittent fever. The abdominal ultrasound was inconclusive and conservative management provided partial improvement. One week before her admission, the transit disorder got worsened with subocclusion.

Initial tests revealed the following vital parameters: blood pressure $90 / 70 \mathrm{mmHg}$, pulse $110 / \mathrm{min}$, respiratory rate $25 / \mathrm{min}$, and temperature $39.1^{\circ} \mathrm{C}$. Examination revealed a firm, mobile, and painful swelling in the epigastric area. There was tenderness in the right iliac fossa and enteric contents through a dehiscence of the laparotomy wound. Blood test revealed an anemia ( $\mathrm{Hb} 8 \mathrm{gm} / \mathrm{dL}$ ) and a high WBC count of $18000 / \mathrm{mm}^{3}$. Gastrografin enema showed a partially obstructing mass in the transverse colon and a fistula tract toward the right iliac fossa (Figure 1). Computed Tomography (CT) scan revealed low density, heterogeneous spongiform mass obstructing the colonic lumen (Figure 2). Emergent laparotomy showed multiple abscesses, and a tumor of the transverse colon. While performing colectomy we found a large abdominal swab $(30 / 30 \mathrm{~cm})$ that had migrated to the colon lumen (Figure 3). In addition, there was a complex enterocutaneous fistula involving the ileum and the ascending colon. The operation ended by colostomy and ileostomy. When reviewing the previous operation report, we found out that there had been a textile count procedure. The 


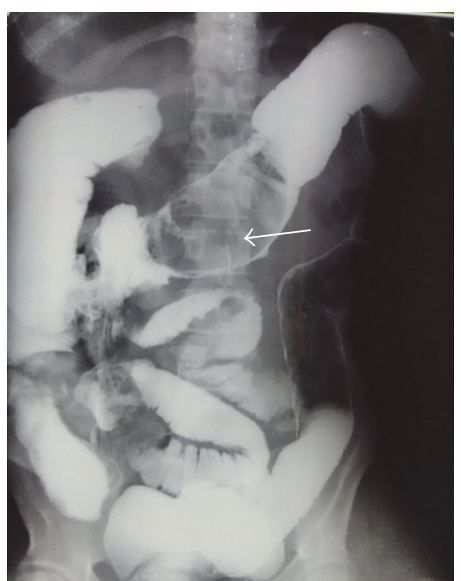

FIGURE 1: Gastrografin enema showing partially obstructing mass (textiloma) in transverse colon lumen with contrast leakage.

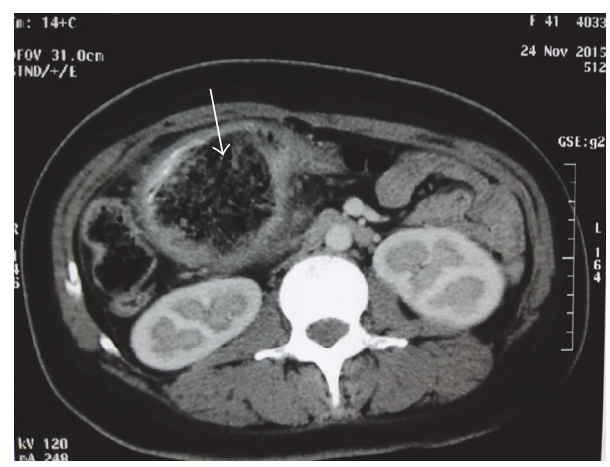

FIGURE 2: Injected abdomen CT scan showing limited spongiform mass with calcifications (textiloma) in the colon lumen.

postoperative period was marked by a persistent fever and a resurgence of enterocutaneous fistula on the seventh day. The death occurred on the ninth day by septic shock.

\section{Discussion}

The retention foreign bodies cause many problems, both diagnostically and therapeutically. Textiles are the most frequent foreign bodies. Their frequency varies between 1/1000 and $1 / 10000$, but this incidence is probably underestimated because of the medicolegal problems associated with these accidents reports [3]. Textile in the abdomen causes a foreign body reaction with exudation or encapsulating granuloma with intestinal adhesion [2]. Exudative reactions may be accompanied with secondary infection and early clinical manifestations while granulomatous reactions can remain asymptomatic for long periods of time. Transmural migration is a rare complication. Zantvoord et al. [4] found 64 cases in the international literature that were mainly located in the ileum between 1960 and 2007. A colic migration as we noticed is even rarer. It may be a secondary location from the ileum via internal fistula [5] or by intestinal peristalsis [6].

Migration occurs followed by an inflammatory necrosis of the intestinal wall and/or an excessive pressure exerted

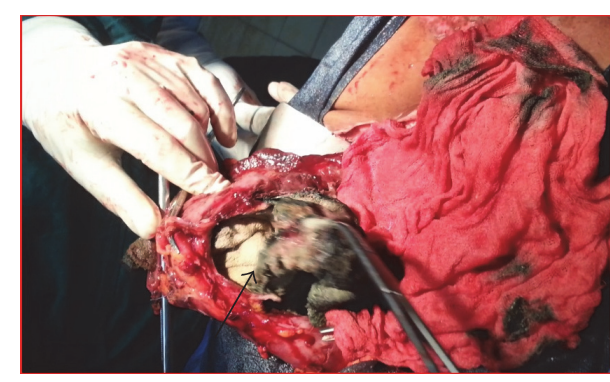

FIGURE 3: Image of perforated colon and abdominal swab in its lumen.

by granuloma around textiloma on the intestinal wall $[3,5]$. No risk factor of transmural migration is identified in the literature. The risk is present from the 10th postoperative week and remains theoretically permanent and even increasing with time [4]. Complications associated with migration are occlusion and internal fistulas $[3,5,7]$. It is uncommon that enterocutaneous fistula follows textiloma transmural migration as we noticed [8]. We believe that the large size of the gauze swab found could be an impediment to a sealed transmural migration as it is used to happen. A timely diagnostic of the textiloma could have helped to avoid such a complication. Plain abdominal radiograph is useful if textiles are marked and not damaged [1]. In ultrasonography, textiloma is suggested by a hypoechoic tumor with a hyperechoic rim and posterior shadowing [7]. CT scan typically shows a well limited hypodense tumor or a spongiform aspect with heterogeneous density including calcifications and air bubbles [9]. Despite that fact, the differential diagnosis with fecal impaction, tumors, and postoperative abscesses can be a challenging situation [6].

The natural course of transmural migration is exceptionally good with the expulsion of textiloma by the anus [4]. In most cases, surgical intervention is required with a bowel resection or enterotomy according to the local conditions [5-8]. The postoperative course is usually good. The death in our case was related to the delay in diagnosis which led to poor local conditions, dissection difficulties, subsequent fistula, and uncontrolled sepsis. This poor outcome recalls the importance of prevention. It is interesting that the postoperative count of textile in our observation was declared complete as in $88 \%$ of textilomas cases [10]. This shows that negligence is not the only aspect of the issue but also prevention must take into account the risk of human error. There are many tools to minimize the risks: exclusive use of marked gauze swabs, systematic X-ray, radio frequency, and bar codes [1]. The use of these instruments is an additional cost; however, the huge legal fees associated with textilomas could be prevented. Where there is lack of resources, systematic textile count remains the only means. Efficient and simple protocols of textiles count must be put in place to increase its reliability.

\section{Conclusion}

Abdominal textilomas are responsible for significant morbidity that can lead to the death of patients. These situations 
involve the entire responsibility of the surgeon, so necessary measures ought to be taken to prevent it.

\section{Consent}

The authors got written consent of the patient relatives to publish this study and the appended images.

\section{Competing Interests}

The authors declare that there is no conflict of interests regarding the publication of this study.

\section{References}

[1] G. H. Sakorafas, D. Sampanis, C. Lappas et al., "Retained surgical sponges: what the practicing clinician should know," Langenbeck's Archives of Surgery, vol. 395, no. 8, pp. 1001-1007, 2010.

[2] S. Wattanasirichaigoon, "Transmural migration of a retained surgical sponge into the intestinal lumen: an experimental study," Journal of the Medical Association of Thailand, vol. 79, no. 7, pp. 415-422, 1996.

[3] R. J. Cruz Jr., L. F. Poli De Figueiredo, and L. Guerra, "Intracolonic obstruction induced by a retained surgical sponge after trauma laparotomy," Journal of Trauma, vol. 55, no. 5, pp. 989991, 2003.

[4] Y. Zantvoord, R. M. F. Van Der Weiden, and M. H. A. Van Hooff, "Transmural migration of retained surgical sponges a systematic review," Obstetrical and Gynecological Survey, vol. 63, no. 7, pp. 465-471, 2008.

[5] E. Margonis, D. Vasdeki, A. Diamantis, G. Koukoulis, G. Christodoulidis, and K. Tepetes, "Intestinal obstruction and ileocolic fistula due to intraluminal migration of a gossypiboma," Case Reports in Surgery, vol. 2016, Article ID 3258782, 4 pages, 2016.

[6] L. Camera, M. Sagnelli, P. Guadagno et al., "Colonic perforation by a transmural and transvalvular migrated retained sponge: multi-detector computed tomography findings," World Journal of Gastroenterology, vol. 20, no. 15, pp. 4457-4461, 2014.

[7] S. C. Sistla, A. Ramesh, V. S. Karthikeyan, D. Ram, S. M. Ali, and R. V. S. Subramaniam, "Gossypiboma presenting as coloduodenal fistula-report of a rare case with review of literature," International Surgery, vol. 99, no. 2, pp. 126-131, 2014.

[8] H. M. Govarjin, M. Talebianfar, F. Fattahi, and M. E. Akbari, "Textiloma, migration of retained long gauze from abdominal cavity to intestine," Journal of Research in Medical Sciences, vol. 15, no. 1, pp. 54-57, 2010.

[9] H. Ersoy, Ö. Barutçu Saygili, and T. Yildirim, "Abdominal gossypiboma: ultrasonography and computerized tomography findings," Turkish Journal of Gastroenterology, vol. 15, no. 1, pp. 65-66, 2004.

[10] A. A. Gawande, D. M. Studdert, E. J. Orav, T. A. Brennan, and M. J. Zinner, "Risk factors for retained instruments and sponges after surgery," The New England Journal of Medicine, vol. 348, no. 3, pp. 229-235, 2003. 


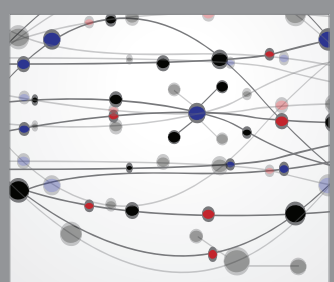

The Scientific World Journal
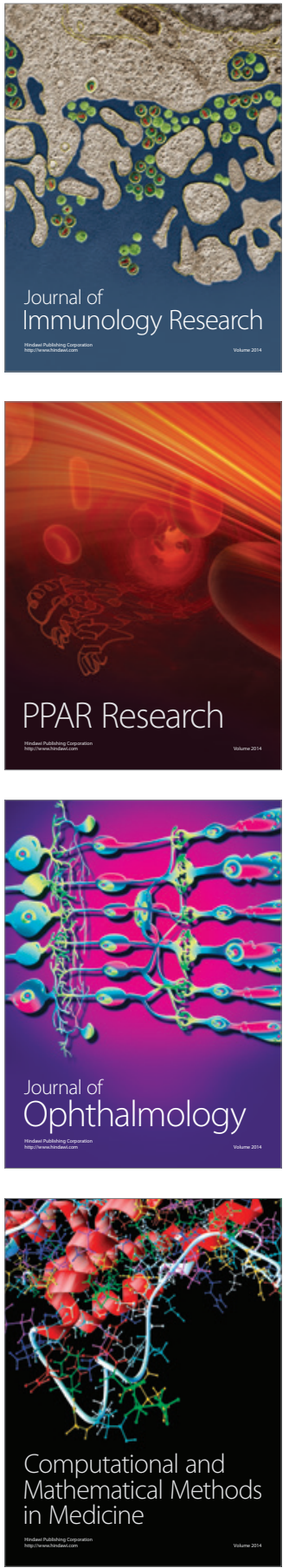

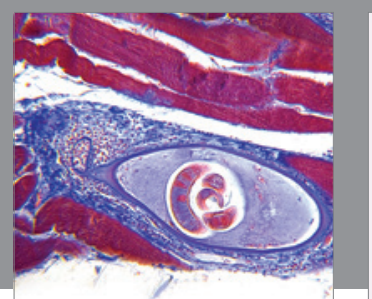

Gastroenterology Research and Practice
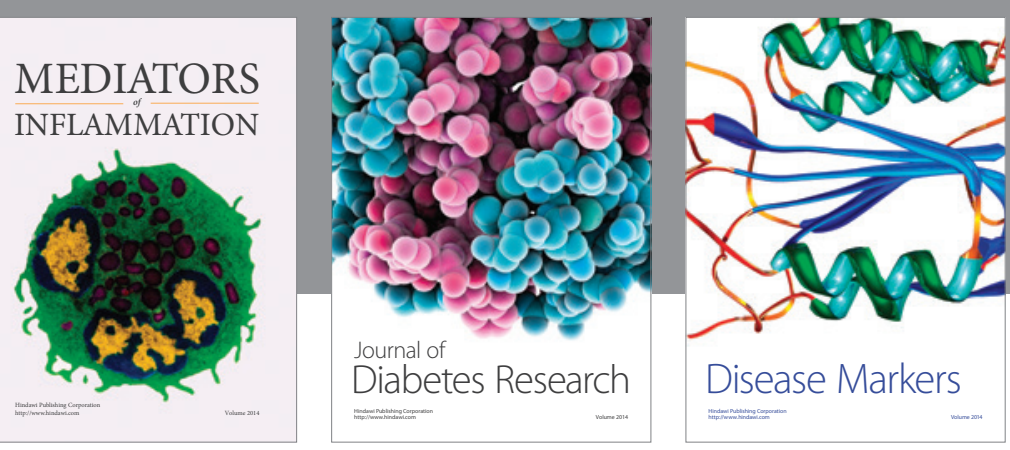

Disease Markers

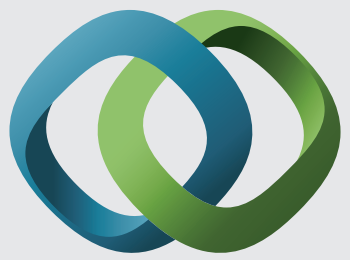

\section{Hindawi}

Submit your manuscripts at

https://www.hindawi.com
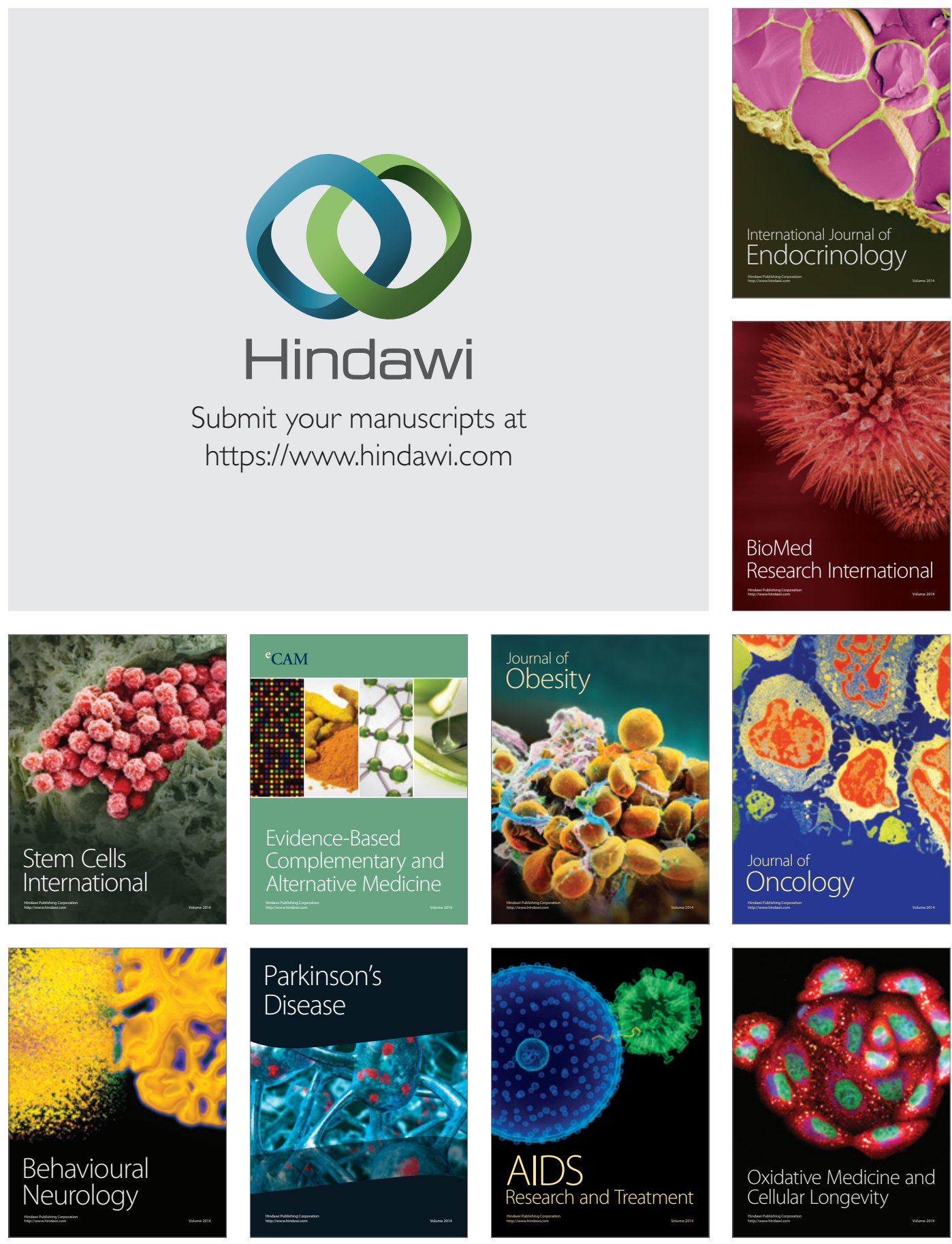